\title{
For the Further Development of the International Journal of Hematology
}

\author{
Takashi Uchiyama and Toshio Suda
}

Int J Hematol. 2006;83:95. doi: 10.1532/IJH97.06004

(C)2006 The Japanese Society of Hematology

\section{Past Editor-in-Chief, Takashi Uchiyama, MD}

Four years have passed since I took over as Editor-in-Chief of the International Journal of Hematology from Dr. Yataro Yoshida. During this period, hematology has made great advances not only in basic research to elucidate the mechanism of blood homeostasis and hematological disorders, but also in clinical research to care for and cure the diseases. IJH has been developing steadily through an effort to publish the papers that contribute to such progress in hematology.

To make further development in the journal, I resign as Editor-in-Chief and am pleased to introduce the new Editorin-Chief, Toshio Suda. I am confident that he will contribute greatly to pushing $\mathrm{IJH}$ forward and providing our readers with the very best, up-to-date information in hematology. In addition, members of the associate editors have been replaced with new ones, and the system of Deputy Editor was abolished.

\section{Editor-in-Chief, Toshio Suda, MD}

From this issue forward, I will serve as Editor-in-Chief for the International Journal of Hematology, which welcomes articles and concise reviews in basic and clinical hematology. IJH has a very long history of publishing some of the leading research in hematology. This journal covers all aspects of the field, namely:

Erythrocytes, Leukocytes \& Hematopoiesis

Hemostasis, Thrombosis \& Vascular Biology

Hematological Malignancies

Transplantation \& Cell Therapy
I thank the former Editor-in-Chief, Dr. Takashi Uchiyama, for his significant efforts on behalf of the members of IJH. He made a significant contribution to this journal by developing the section "Progress in Hematology" that was initially introduced by Dr. Yataro Yoshida. I will extend this section by integrating into it research fields highly relevant to hematology. Examples of such fields are the cell biology of stem cells and cancer cells and clinical research in inflammation, cancer, and thrombosis. Moreover, papers reporting results of clinical trials will also be included to accelerate the development of translational research.

Many journals compete for high impact manuscripts, and very original papers focusing on challenging issues are not always published in the so-called "high profile journals." In the field of hematopoiesis, for example, some epoch-making papers, such as the discovery of stem cells and the establishment of in vitro colony assay, have been published in journals such as Radiation Research (1961) and the Australian Journal of Experimental Medicine (1969), respectively. It is my hope that IJH will become such a vehicle for critical publications. To facilitate rapid publication of research, our editorial board will make every effort to complete the review process for papers as quickly as possible (optimally, 2 weeks). Also, we will foster communication among researchers through meeting reviews and letters to the editor. I hope that this journal will contribute significantly to research in modern hematology and be recognized as one of the outstanding journals in the field. 